\title{
Las niñas y adolescentes
}

\author{
Pablo Enrique Uribe*; Rodrigo León Mesa*, Fabio Sánchez**
}

\section{RESUMEN:}

OBJETIVO: Evaluar en una consulta especializada de ginecología para niñas y adolescentes, las entidades más frecuentes por la cual consultan y con el fin de orientar en el futuro programas de prevención y tratamiento en pacientes en este grupo de edad, como también elaborar programas de educación médica para los futuros especialistas en ginecología y pediatría para que manejen con propiedad estas patologías.

MATERIAL Y METODOS: Se hizo un estudio descriptivo prospectivo en la consulta externa de .............. y bajo la supervisión del departamento de obstetricia y ginecología de desde agosto de 1993 a diciembre de 1994 durante 4 horas semanales.

Luego de una entrevista, examen clínico y exámenes de laboratorio específicos para cada una de las patologías sospechadas, posteriormente se obtuvo un diagnóstico específico. Todos los datos fueron consignados en un formulario precodificado y las variables analizadas en un computador 386 y en el programa de Epiinfo.

RESULTADOS: Se atendieron un total de 113 niñas, siendo el $92.9 \%$ de la ciudad de y del área metropolitana. EI $70 \%$ eran adolescentes y el $30 \%$ niñas menores de 9 años.

Cuando se evalúan los diagnósticos más frecuentes, el síndrome de ovario androgénico (SOA) alcanza el $15.9 \%$ y la vulvo vaginitis inespecífica $12.4 \%$ se obtuvieron un total de 36 diagnósticos.

Cuando se clasifican los diagnósticos en 5 grandes grupos, el $46 \%$ correspondió a las enfermedades endocrinas. $28.3 \%$ a infecciones vulvo vaginales, $6.2 \%$ a trastornos benignos de la mama, $4.4 \%$ dolor pélvico y un $15 \%$ a misceláneas.

Se hace una evaluación de los problemas endocrinos más frecuentes, donde predomina la hiperandrogenemia y de éstas el SOA tiene un $34.6 \%$. En cuanto a las manifestaciones clínicas más frecuentes de la hiperandrogenemia fue el acné con un $65.21 \%$, los trastornos del ciclo con $21.73 \%$, el hirsutismo con un $13.84 \%$ y la obesidad con un $8.6 \%$.

DISCUSION: es necesario que en todos los hospitales del país se continúen creando programas de atención ginecológica a la niña y a la adolescente, su morbilidad es mayor que lo que realmente se piensa, pues en ocasiones ésta necesidad es suplida por el pediatra. El hacer una consulta especializada en ésta época nos permite prevenir mayores complicaciones en la edad adulta y al mismo tiempo brindarles asesoría y educación a éste grupo de alto riesgo.

El papel del ginecólogo no es solamente dedicarse a la edad reproductiva de la mujer, sino abarcar todo su proceso biológico integralmente desde la niñez, adolescencia, vida adulta, climaterio y senectud; siendo las dos primeras etapas las que ofrecen mayor posibilidad de prevención y garantizan mejores condiciones de vida en las próximas etapas.

\section{Introducción}

Es importante que en nuestro medio, se inicien programas dirigidos al estudio del desarrollo ginecológico normal y patológico en la niña y en la adolescente, con el fin de llenar los vacíos académicos de la formación médica y sirvan a la vez de orientación en la programación de futuros estudios prospectivos que permitan delinear políticas en salud dirigidas a una atención más integral de este grupo etáreo.

La población infantil y especialmente la adolescente no está exenta de sufrir enfermedades ginecológicas, muchas de estas patologías son manejadas por pediatras y cirujanos infantiles, por lo cual se hace necesaria la participación del ginecólogo en el grupo interdisciplinario con el fin de orientar muchas de las patologías existentes.

\footnotetext{
* Residentes de Tercer año.

** $\quad$ Profesor Titular.
}

Diversas instituciones a nivel nacional abordan el tema de la adolescente, principalmente con respecto al embarazo, pero faltan estudios descriptivos que orienten sobre la frecuencia de los diversos trastornos ginecológicos de la juventud y de la niñez.

Es por esto que se programó en nuestro Departamento un estudio descriptivo prospectivo en niñas y adolescentes para que sirvan de base para futuros estudios analíticos.

\section{Materiales y métodos}

La población estudiada son niñas y adolescentes con trastornos ginecológicos, que acudieron a la consulta especializada del Hospital Infantil....., agosto de 1993 a diciembre de 1994. La información se obtuvo por entrevista directa, examen clínico y exámenes de laboratorio complementarios dirigidos a obtener un diagnóstico específico, en forma prospectiva. 
Los trastornos endocrinos se diagnosticaron por medio de criterios clínicos complementados con determinaciones hormonales de acuerdo a la entidad clínica: Estradiol, Dehidro epiandrosterona sulfato, Testosterona libre, $3 \alpha$ Androstenodiol glucorónido, Prolactina $17 \alpha$ Hidroxiprogesterona, Tirotropina, Tiroxina y Triyodotironina: y comparados con los valores estándar del laboratorio del Hospital San Vicente de Paúl.

El flujo vaginal se estudió por medio de Gram y directo con el fin de confirmar el diagnóstico y en casos especiales se solicitaron cultivos.

Para el diagnóstico del dolor pélvico y la patotogía mamaria, se tuvo en cuenta la historia clínica, exámenes paraclínicos como su respuesta al tratamiento médico.

Con el fin de evitar la repetición de diagnósticos se tomó el más relevante en cada una de las pacientes.

Los datos obtenidos fueron consignados en un formato precodificado de historia clínica y las variables analizadas en un computador 386 por medio del programa Epi-Info.

\section{Resultados}

Se estudiaron 113 pacientes menores de 20 años que consultaron al Servicio de Ginecología de la Niña y la Adolescente, en el Hospital Infantil.........., quienes procedían de la ciudad....... el $74.3 \%$ y el $92.9 \%$ del Area Metropolitana que es el área de mayor influencia del Hospital (tabla 1).

\section{Tabla 1 \\ CLASIFICACION DE LAS NIÑAS Y LAS ADOLESCENTES CON RELACIÓN AL LUGAR DE PROCEDENCIA}

\begin{tabular}{|lcc|}
\hline Procedencia & Pacientes & Porcentaje \\
\hline Municipio & 84 & 74.3 \\
Municipios del Area & 21 & 18.6 \\
Metropolitana & 7 & 6.2 \\
Otros Municipios del & 1 & 0.9 \\
Departamento & 113 & 100 \\
Otros Departamentos & & \\
\hline TOTAL & & \\
\hline
\end{tabular}

Con respecto a la edad, se incluyeron pacientes menores de 20 años. De las pacientes estudiadas, el 30\% son menores de nueve años y el $70 \%$ adolescentes entre $10 \mathrm{y}$ 20. La edad predominante se presentó entre 13 y 15 años con un $31,8 \%$ y con una media de 12,1 y una DS de $\pm 4,85$. (tabla 2).

Cuando se analizan los diagnósticos obtenidos, se aprecia cómo el síndrome de ovario androgénico y la vulvovaginitis inespecífica, son los más frecuentes y representan el 15,9 y el $12,4 \%$ respectivamente. Pero cuando se aglutinan varias diagnósticos de acuerdo con su etiología, la vulvovaginitis por diferentes causas: inespecífica, por gardnerella, candidiasis vaginal, herpes genital, alcanza un mayor porcentaje $23,8 \%$, en 27 pacientes estudiadas, los demás diagnósticos tienen una menor frecuencia. (Tabla 3 ).
Tabla 2

DISTRIBUCION PORCENTUAL DE LAS NIÑAS Y ADOLESCENTES CON RELACION A LA EDAD

\begin{tabular}{|lccc|}
\hline Edad & Pacientes & $\%$ & \% \\
\hline$<1$ & 1 & 0.9 & 0.9 \\
$1-3$ & 0 & 7.9 & 8.8 \\
$4-6$ & 12 & 10.6 & 19.4 \\
$7-9$ & 12 & 10.6 & 30.0 \\
$10-12$ & 20 & 17.7 & 47.7 \\
$13-15$ & 36 & 31.8 & 79.5 \\
$16-18$ & 20 & 17.7 & 97.2 \\
$19-20$ & 3 & 2.8 & 100.0 \\
\hline TOTAL & 113 & 100.0 & 100.0 \\
\hline
\end{tabular}

Tabla 3

DISTRIBUCION DE LOS DIFERENTES DIAGNOSTICOS OBTENIDOS

\begin{tabular}{|c|c|c|c|}
\hline \# & Diagnóstico & Pacientes & Porcentaje \\
\hline 1 & Síndrome de ovario androgénico & 18 & 15,9 \\
\hline 2 & Vulvo Vaginitis Inespecífica & 14 & 12,4 \\
\hline \multirow[t]{2}{*}{3} & Vaginosis por Gardnerella & & \\
\hline & Vaginalis & 9 & 7,9 \\
\hline 4 & Pubertad Precoz & 8 & 7,1 \\
\hline 5 & Pubertad Normal & 7 & 6,2 \\
\hline 6 & Hemorragia Uterina Anormal & 6 & 5,3 \\
\hline 7 & Pubertad Retardada & 5 & 4,4 \\
\hline 8 & Candidiasis & 4 & 3,5 \\
\hline 9 & Endometriosis & 3 & 2,6 \\
\hline 10 & Gonorrea & 2 & 1,8 \\
\hline 11 & Enfermedad pélvica inflamatoria & 2 & 1,8 \\
\hline 12 & Mastodinia cíclica & 2 & 1,8 \\
\hline 13 & Fibroadenoma mamario & 2 & 1,8 \\
\hline 14 & Disgenesia gonadal & 2 & 1,8 \\
\hline 15 & Hiperplasia suprarrenal & 2 & 1,8 \\
\hline \multirow[t]{2}{*}{16} & Disfunción hipotálamo-hipófisis & & \\
\hline & $\mathrm{x}$ bajo peso & 2 & 1,8 \\
\hline 17 & Violación & 2 & 1,8 \\
\hline 18 & Planificación familiar & 2 & 1,8 \\
\hline 19 & Mal definición sexual & 2 & 1,8 \\
\hline 20 & Herpes genital & 1 & 0,9 \\
\hline 21 & Cuerpo extraño intravaginal & 1 & 0,9 \\
\hline 22 & Colon irritable & 1 & 0,9 \\
\hline 23 & Quiste torcido de Ovario & 1 & 0,9 \\
\hline 24 & Síndrome de tensión premenstrual & 1 & 0,9 \\
\hline 25 & Nódulo mamario & 1 & 0,9 \\
\hline 26 & Mastopatía fibroquística & 1 & 0,9 \\
\hline 27 & Hipoplasia mamaria & 1 & 0,9 \\
\hline 28 & Hipogonadismo-hipogonadotropo & 1 & 0,9 \\
\hline 29 & Menopausia quirúrgica & 1 & 0,9 \\
\hline 30 & Hipotiroidismo & 1 & 0,9 \\
\hline 31 & Embarazo & 1 & 0,9 \\
\hline 32 & Himen íntegro & 1 & 0,9 \\
\hline 33 & Infección urinaria & 1 & 0,9 \\
\hline 34 & Capuchón redundante clítoris & 1 & 0,9 \\
\hline 35 & Post parto & 1 & 0,9 \\
\hline \multirow[t]{2}{*}{36} & Amenorrea secundaria & 1 & 0,9 \\
\hline & TOTAL & 113 & 100.0 \\
\hline
\end{tabular}


Los anteriores diagnósticos se agruparon para su análisis en cinco categorías: alteraciones endocrinas, enfermedades infecciosas, dolor pélvico, alteraciones benignas de la glándula mamaria y misceláneas.

Se observa el predominio de las alteraciones endocrinas, en 52 pacientes (46\%) y en segundo lugar las enfermedades infecciosas, con 28.3 (32 pacientes), estos dos grupos abarcan el $74.3 \%$ de los diagnósticos de la consulta, siendo las enfermedades endocrinas predominantes en el grupo de adolescentes en tanto las infecciones vulvovaginales en la población infantil.

Las enfermedades infecciosas vulvovaginales ocuparon el segundo lugar, luego de las enfermedades endocrinas con 32 pacientes $(28.3 \%)$ entidad que predomina más en las niñas, 21 pacientes (18.5\%). El diagnóstico más frecuente correspondió a la vulvovaginitis inespecífica, pues no se encontró un germen causal y en ocasiones se aisló flora normal, en segundo lugar estuvo la gardnerella con un $26.4 \%$.

La categoría Miscelánea comprende diferentes diagnósticos que no son posibles ordenarlos en un grupo específico tales como: pubertad normal, embarazo, postparto, acceso carnal violento, cuerpo extraño intravaginal, himen intacto, capuchón del clítoris redundante (tabla 4).

En las enfermedades endocrinas predominó el síndrome de ovario androgénico $18(34,6 \%)$ le siguen la pubertad precoz $8(15,4 \%)$ y la hemorragia uterina anormal, 11,5\%. Hallazgo que resulta más importante cuando se analiza en conjunto todas las pacientes con hiperandrogenemia, $23(44.2 \%)$ del total de pacientes con alteración endocrina y $20,4 \%$ del número total de pacientes (tabla 5).

E1 $40,35 \%$ de las 52 pacientes estudiadas con alteraciones endocrinas, presentaron como motivo de consulta, manifestaciones de origen hiperandrogénico, siendo el síndrome de ovario androgénico el más frecuente con $78,2 \%$. Las demás entidades clínicas tienen una menor frecuencia (tabla 6).

Cuando se evalúan las manifestaciones hiperandrogénicas aparece como la mayor expresión el acné en un porcentaje muy alto de un $65,2 \%$ y las alteraciones del ciclo sólo se presenta en una quinta parte de las pacientes y ya empieza a presentarse en ésta época el hirsutismo como manifestación de hiperandrogenemia en un grupo demasiado joven para presentar esta manifestación, sólo dos pacientes presentaron manifestaciones de obesidad al momento de la consulta (Tabla 7).

Cinco pacientes $(4,42 \%)$ de la población estudiada y el $6,3 \%$ del grupo adolescente consultó por dolor pélvico, correspondiente tres de ellos a dismenorrea primaria, una a quiste torcido de ovario, quien fue intervenida quirúrgicamente y una con colon irritable. A las pacientes con dismenorrea primaria se les inició terapia con AINES antes de indicárseles una laparoscopia diagnóstica.

Ocho pacientes consultaron por pubertad precoz, seis de ellas (75\%) entre cinco y ocho años y dos menores de cuatro años, siendo una menor de un año.

Los demás parámetros que marcan el desarrollo puberal tal como pubarquia y telarquia no fueron cuantificadas, máxime que la información era muy subjetiva.
Tabla 4

DISTRIBUCION DE LOS DIFERENTES DIAGNOSTICOS CLASIFICADOS EN CINCO GRUPOS

\begin{tabular}{|lcc|}
\hline Diagnóstico & Pacientes & Porcentaje \\
\hline Alteraciones Endocrinas & 52 & 46,0 \\
Enfermedades Infecciosas & 32 & 28,3 \\
Enfermedad Benigna de la mama & 7 & 6,2 \\
Dolor Pélvico & 5 & 4,4 \\
Misceláneas & 17 & 15,0 \\
\hline TOTAL & 113 & 100,0 \\
\hline
\end{tabular}

Tabla 5

DISTRIBUCION PORCENTUAL DE LAS ALTERACIONES ENDOCRINAS

\begin{tabular}{|llcc|}
\hline$\#$ & Entidades & Pacientes & Porcentajes \\
\hline 1 & Sínd. Ovario Androgénico & 18 & 34.6 \\
2 & Pubertad Precoz & 8 & 15.4 \\
3 & Hemorragia Uterina Anormal & 6 & 11.5 \\
4 & Pubertad Retardada & 5 & 9.6 \\
5 & Disgenesia gonadal & 2 & 3.8 \\
6 & Hipertricosis & 2 & 3.8 \\
7 & Hiperplasia Suprarrenal & 2 & 3.8 \\
8 & Disfunción Hipotálamo & & \\
& Hipofisiaria x Bajo Peso & 2 & 3.8 \\
9 & Maldefinición Sexual & 2 & 3.8 \\
10 & Sínd. Tensión Premenstrual & 1 & 1.9 \\
11 & Hipogonadismo Hipogonadotropo & 1 & 1.9 \\
12 & Menopausia Quirúrgica & 1 & 1.9 \\
13 & Hipotiroidismo & 1 & 1.9 \\
14 & Amenorrea Secundaria & 1 & 1.9 \\
\hline TOTAL & 52 & 100.0 \\
\hline
\end{tabular}

Tabla 6

DISTRIBUCION PORCENTUAL DE LOS DIFERENTES DIAGNOSTICOS DE HIPERANDROGENEMIA

\begin{tabular}{|lcc|}
\hline Diagnóstico & Pacientes & Porcentaje \\
\hline Sínd. Ovario Androgénico & 18 & 78.2 \\
Hipertricosis & 2 & 8.7 \\
Hiperplasia Suprarrenal & 2 & 8.7 \\
Hipotiroidismo & 1 & 4.3 \\
\hline TOTAL & 23 & 100.0 \\
\hline
\end{tabular}

Tabla 7

DISTRIBUCION DE LAS MANIFESTACIONES CLINICAS EN PACIENTES CON HIPERANDROGENEMIA

\begin{tabular}{|lcc|}
\hline Manifestaciones & Pacientes & Porcentaje \\
\hline Acné & 15 & 65,21 \\
Oligomenorrea & 5 & 21,73 \\
Hirsutismo & 3 & 13,84 \\
Obesidad & 2 & 8,60 \\
\hline TOTAL & 23 & 100,0 \\
\hline
\end{tabular}


Con respecto a la patología mamaria se estudiaron siete pacientes $(6.2 \%)$ o $8.8 \%$ de la población adolescente, donde aún no se encuentra una patología dominante: dos pacientes con mastodinia, tres con fibroadenoma, una con mastopatía fibroquística y una con hipoplasia mamaria.

Del grupo de 58 adolescentes estudiadas, sólo 22 $(37.9 \%)$ de las pacientes, informaron que planificaban con algún método así: anticonceptivos 14 , siete con preservativos y una con DIU.

De las 58 adolescentes,. $12(20.68 \%)$ tienen historia de uno o dos embarazos así: nueve adolescentes con un embarazo y tres con dos.

\section{Discusión}

Al iniciar la consulta Ginecológica de la Niña y de la Adolescente como parte del Servicio de Obstetricia y Ginecología, era necesario conocer algunos datos generales de la población consultante (1-3), sinembargo fue escasa la afluencia de pacientes a ese servicio especializado, debido a que la mayoría de ellas continuaban bajo control con el pediatra o el médico general y sólo se referían a nuestro servicio aquellas pacientes que tenían algún grado de dificultad en el diagnóstico o en el tratamiento ginecológico, proceso que viene modificándose una vez se reconozca el servicio y se trabaje en forma interdisciplinaria.

Como era de esperarse, la mayoría de las pacientes que consultaron provenían de la ciudad de Medellín y del área Metropolitana pero en la medida en que el servicio tenga mayor difusión se podría ampliar su área de influencia, y además se tiene la mira de convertirlo en un centro multiplicador para entrenamiento de residentes en Obstetricia y Ginecología.

El mayor número de pacientes estudiadas correspondió al grupo de las adolescentes $70 \%$, sin embargo es importante llamar la atención que el 30\% restante de las consultantes eran niñas, muchas de ellas con historia de recidivas y sometidas a múltiples tratamientos, antes de asistir al ginecólogo, como ocurrió con las niñas que consultaron por leucorreas.

Cuando se analizan los diferentes diagnósticos de la población estudiada, surge el síndrome de ovario androgénico como el más frecuente, 52 pacientes (46\%), como consecuencia de los cambios endocrinos que ocurren en la mujer durante esta época de la vida $(4,5)$ y con sus manifestaciones de trastornos del ciclo, piel grasosa, acné y obesidad; la vulvovaginitis inespecífica alcanza el segundo lugar, con 32 pacientes $(28,3 \%)$; la patología mamaria el tercer lugar con un $6,2 \%$ y el dolor pélvico un $(4,4 \%)$, el cual fue el de menor frecuencia.

En el análisis de estos resultados es importante apreciar como la infección vulvovaginal es más frecuente en el grupo de niñas, y con alto grado de recidiva como consecuencia no sólo de las características físico-químicos de la vagina atrófica, con ausencia de glicógeno y $\mathrm{pH}$ alcalino, sino también por la coexistencia de malos hábitos higiénicos no sólo en el baño, luego de orinar y defecar, sino también por la exposición y el contacto de los genitales a la humedad y a la contaminación del suelo (6).
Para que el tratamiento en estas niñas sea exitoso es necesario prescribir estrógenos en dosis bajas durante un mes, e iniciar el tratamiento específico unos 15 días después del primero una vez se hallan obtenido modificaciones fisicoquímicas y citologías en la vagina, además se debe asociar a un manejo adecuado en la higiene personal, como también evitar la contaminación de sus genitales.

Las infecciones de transmisión sexual se presentaron en un porcentaje realmente importante $4,5 \%$ de la población estudiada, porcentaje muy alto y preocupante en una población de alto riesgo y de bajo nivel socioeconómico, muchas de ellas con alta probabilidad de presentar embarazos no deseados, infertilidad, sufrir infecciones pélvicas y aún someterse a anexo-histerectomías, como ocurrió con una de las pacientes.

Individualmente en los diagnósticos, también el síndrome de ovario androgénico ocupó el primer lugar con 18 pacientes $(15,9 \%)$ del total, siendo más frecuente en el grupo de las adolescentes (4) y cuyas manifestaciones físicas son muy evidentes, siendo la principal manifestación el acné, en donde cerca del $65,2 \%$ de las 23 pacientes hiperandrogénicas lo sufrían al momento de la consulta y en una época que se considera la plenitud de la vida, este fenómeno compromete la autoestima de las pacientes y llega al punto de que muchas de ellas optan por aislarse, máxime si se asocia a obesidad e hirsutismo (5), es por ello que ésta manifestación no debe considerarse como un proceso normal de la adolescente y es obligación del médico evitar o atenuar la presencia del acné.

Es importante que el médico informe a los adolescentes la importancia que tiene conservar una buena salud, evitar la obesidad y utilizar una terapia precoz dirigida a prevenir estos efectos indeseables, además ilustrarlos sobre el proceso de la entidad y su pronóstico con el fin de que desde la adolescencia se eviten estas manifestaciones indeseables.

Para ello es necesario la conformación de un equipo interdisciplinario constituido por pediatras, ginecólogos, dermatólogos, nutricionistas y aún psicólogos y psiquiatras que enfrenten este problema desde varios puntos de vista, para que diagnostiquen y traten precozmente esta patología y mejoren el pronóstico de estas pacientes.

Llama la atención el número tan bajo de mal definición sexual, debido a que esta patología tradicionalmente ha sido manejada en nuestro medio por los cirujanos, es así como las dos únicas pacientes que estudiamos estaban en la adolescencia.

Se hace necesario que por la complejidad del problema de mal definición sexual (8) sea manejado por un equipo interdisciplinario en donde el pediatra, el ginecólogo y el endocrinólogo estudien el problema básico, y que con el cirujano asuman la conducta quirúrgica, además se asesoren de psicólogos, psiquiatras y terapeutas de familia.

Ocho pacientes $(0.7 \%)$ tuvieron el diagnóstico de pubertad precoz y sólo se trataron con progestágenos (9), con buena respuesta en el sentido de que se frenó el desarrollo de los caracteres sexuales y se controlaron los niveles elevados de estradiol: por la capacidad económica de los padres fue imposible la terapia con análogos de la GnRh. 
Aunque la patología mamaria y el dolor pélvico $(10,11)$ alcanzaron porcentajes muy bajos, no deja de ser preocupante el inicio tan precoz de estas entidades que son tan incapacitantes para la mujer, es por ello que se hace necesario prevenirlas, pues su historia natural tiene una tendencia hacia el agravamiento.

En la dismenorrea de la adolescente la más frecuente es la primaria inducida por prostaglandinas $\mathrm{F}_{2} \alpha$, sin embargo no debe destacarse la endometriosis como causa etiológica además de otras entidades pueden desencadenar dolor pélvico crónico tales como tumores sólidos o quísticos de ovario, es así como las pacientes con síndrome de ovario androgénico pueden sufrir esta patología debido a que los ovarios adquieren un tamaño 2 o 3 veces más que lo normal, se debe destacar también patología de colon.

Se tienen muchas teorías con respecto al origen de la endometriosis, y aunque la teoría del reflujo ha perdido mucha importancia debido a que es un fenómeno que se ha encontrado frecuentemente durante la menstruación en mujeres fértiles y sin historia de endometriosis, sin embargo no se debe destacar esta teoría en pacientes anovulatorias, en quienes las células endometriales que refluyen se encuentran en fase proliferativa, con plena capacidad de reduplicación y de implantación, proceso que no ocurría en mujeres con ciclos ovulatorios donde estas células están en fase secretora, las cuales han perdido su capacidad de implantación y reduplicación.

La patología mamaria, aunque menos frecuente, no deja de ser importante puesto que ya empieza a presentarse en esta edad mastodinias que en ocasiones son incapacitantes principalmente en la época premenstrual, los fibroadenomas aunque escasos no es necesario intervenirlos, sólo se haría en la medida en que sean de gran tamaño o muy sintomáticos. En esta época son frecuentes las consultas por desarrollo mamario: hipoplasia o gigantomastia, que en la medida en que ellos comprometan el estado sicológico y la autoestima de las pacientes, éstas deben ser intervenidas quirúrgicamente previa evaluación de su nivel hormonal y después de habérsele comprobado varios ciclos ovulatorios o haber sido tratada durante varios meses con progestágenos con el fin de asegurarles el mayor desarrollo de la glándula mamaria.

Siendo la incidencia de las enfermedades endocrinas en el grupo de adolescentes, de más del $50 \%$, se hace necesario de que el perfil del médico especialista en adolescentes sea un ginecólogo, con entrenamiento en el área de la endocrinología y aunque en este estudio no se hace muy evidente, es necesario que tenga entrenamiento en genética y embriología, por la complejidad de las patologías de las pacientes tal como la mal definición sexual y las disgenesias gonadales.

Es importante incluir en la consulta de estas pacientes una orientación sobre planificación familiar, debido al mayor riesgo de embarazos no deseados, abortos inducidos o madresolterismo que ocurre durante esta época, es así como un $37.9 \%$ de las adolescentes planificaban, por lo cual nos hace pensar que el porcentaje de adolescentes con vida sexual activa era mucho mayor pero que no lo informaron al momento de la consulta, en muchas ocasiones porque estaban acompañadas por la madre o por un familiar.

Encontrar un 20.68\% de adolescentes embarazadas o sea una de cada 5 consultantes es un porcentaje muy alto en este grupo etáreo, en una población de alto riesgo, de insuficiente preparación intelectual, escasa capacidad económica, sin el apoyo familiar generalmente y sin la madurez sicológica para asumir la responsabilidad que exige la maternidad.

Es necesario que los Departamentos de Obstetricia y Ginecología, organicen servicios especializados en Ginecología Pediátrica y del Adolescente, con el fin de trabajar en forma interdisciplinaria con otras especialidades para el manejo integral de estas patologías.

\section{BIBLIOGRAFIA}

1. Berman RE., Vaughan VC En: Tratado de Pediatría. 12 Edición. México, Interamericana, 1985; 1937.

2. Cusminisky M., Méndez JM. Problemas ginecológicos comunes. En: Maddaleno, M., Munist MM., Silber TJ., Suárez, E. Manual de Medicina de la Adolescencia. Organización Panamericana de la Salud. $1992 ; 250-272$.

3. Ministerio de Salud de Colombia. Atención integral en salud en el adolescente. Bogotá, 1990; 55. (Estudio Nacional de Salud).

4. Henao G. y Mejía JM. Estados de hiperandrogenemia. En: Botero J., Henao G., Jubiz A. Texto Integrado de Obstetricia y Ginecología. Medellín. Cuarta edición, Carvajal S.A. 1989; 622-643.

5. Méndez JM. Hirsutismo. En: De Zeiguer BK. Ginecología infanto juvenil. Buenos Aires, Editorial Panamericana, Segunda edición, 1988; 335-340.

6. De Zeiguer BK., De Zeiguer N. Infecciones del tracto genital inferior. En: De Zeiguer, BK. Ginecología infanto juvenil. Buenos Aires, Editorial Panamericana. Segunda edición, 1988; 149-218.

7. Silber TJ. Enfermedades de transmisión sexual durante la adolescencia. En: Maddaleno M., Munist MM., Suárez E. Manual de Medicina de la Adolescencia. Organización Panamericana de la Salud, 1992; 279-303.

8. Mahoney CP. Trastornos de diferenciación y trastornos sexuales. Endocrinología pediátrica y de adolescentes. Clin. Pediát. N. Am. 1990; 6: 1463-1480.

9. Styne D. y Wheeler MD. Diagnóstico y tratamiento de la pubertad precoz. Endocrinología pediátrica y de adolescentes. Clin. Pediát. N. Am. 1990; 6: 1317-1332.

10. Dubcorsky J. Dolor pelviano. En: De Zeiguer, BK. Ginecología infanto juvenil Buenos Aires, Editorial Panamericana. Segunda edición, 1988; 233-249.

11. Farrel EG., Greydanus DE., Parks DS. Trastornos mamarios en niñas y adolescentes. Ginecología de adolescentes. Clin. Pediát. N. Am. 1989; 3: 647-688.

12. Organización Panamericana de la Salud. La Salud del Adolescente y el joven en las Américas. Publicación científica No. 489. Washington, D.C. $1985 ; 385$. 